\title{
Visuelle Spezifikation des Kerns objektorientierter Applikationen
}

\author{
Gerd Szwillus \\ Universität - GH - Paderborn \\ Fachbereich Mathematik/Informatik \\ Warburger Straße 100 \\ D-4790 Paderborn \\ szwilluseuni-paderborn.de
}

Für die Entwicklung interaktiver, objektorientierter Applikationen hat sich das klassische Seeheim-Modell [Pfaff 85] zur Strukturierung von Benutzungsschnittstellen nicht bewährt. An die Stelle einer zentralen Dialogkontrolle tritt heute typischerweise die Idee einer gemeinsamen Objektwelt von Applikation und Benutzungsschnittstelle ("shared application model") [Dance et al 87]. Allerdings hat dies die Konsequenz, daß die Codeanteile von Applikation und Benutzungsschnittstelle wieder stark durchmischt bzw. auf viele Objekte verteilt werden. Dies läuft grundsätzlich der Forderung nach Trennen der Aufgaben Anwendungsprogrammierung und Schnittstellenprogrammierung entgegen. Das Ziel dieser Arbeit ist es, eine visuelle Notation mit einer zugehörigen Methodik zu entwickeln, die es erlaubt, Applikationen mit komplexen Benutzungsschnittstellen auf der Basis der Architektur "gemeinsamer Objekte" zu entwerfen. Durch eine klare Spezifikation der Schnittstelle zwischen Applikation und Benutzungsschnittstelle, des hier so genannten Objektkerns, wird es möglich, die Entwicklung beider Anteile streng voneinander zu trennen.

\section{Einführung}

Die visuelle Darstellung des Objektkerns abstrahiert von technischen Eigenschaften einer konkreten objektorientierten Programmiersprache und besitzt andererseits genug Ausdruckskraft, um die - für den Zweck erforderlichen - Beziehungen festzulegen. Grundsätzlich ist dieser Spezifikationsschritt Teil einer Phase der objektorientierten Analyse des Anwendungsproblems - wie etwa mit OBA [Rubin und Goldberg 92] - konzentriert sich festgengs auf eine Oberflächensicht auf die Applikation: Die Teilobjektwelt, die hier In dieser Phard, beschränkt sich auf die für die Benutzung des Systems relevanten Objekte. und der Entwickler der Beng arbeiten der Entwickler der Applikation (AP-Entwickler) "Vertragsgrundlage" der Benutzungsschnittstelle (BS-Entwickler) zusammen, um diese anschließend die Teir dann weitgehend separaten Tätigkeiten festzulegen. Beide können gemeinsamen Kern aber nabhängig voneinander nur noch erweitem, den geschehen auf beid aber nicht mehr ohne explizite Absprache verändern. Erweiterungen Zwischen den beiden Seiten durch das Hinzufügen von Klassen, Methoden und Attributen. Attribute und Meiden Entwicklern besteht Einigkeit darüber, daß es genau die Klassen, ggf. veränderbar oden des Objektkerns sind, die bei der Benutzung sichtbar, benutzbar und angeboten - alle an mussen. AusschlieBlich diese Komponenten werden dem Benutzer dieser Klassen in der Benutzungsschtbar. Etwas genauer gesagt heißt das, daß nur Objekte Attribute die Darstellung beeinfluschnittstelle repräsentiert werden, daß nur die erwähnten Benutzer zur Ausführung zur Verfügung sönnen und daß nur die angeführten Methoden dem 


\section{Die Objektkern-Notation}

Objekte des objektorientierten Paradigmas werden als autonom handeinde Agenten aufgefaßt, die sich durch Identität, Gedächtnis und Verhalten [Booch 91] auszeichnen. Dieses Konzept legt eine Darstellung von Objekten bzw. Klassen als räumlich (optisch) zusammenhängende Stnkturen nahe, wie bereits in ähnlichen derartigen Notationen ausgeführt - siehe etwa [Booch 91], [Wirfs-Brock, Wilkerson und Wiener 90], [Coad und Yourdon 91], [Felser 92]. Die Objektkern-Notation stellt Enthaltenseins-Beziehungen (wie etwa in Klassen definierte Methoden) durch Verwendung von graphischem "Ineinanderschachteln" und VerweisBeziehungen (wie etwa die Vererbungsbeziehung) durch Linien bzw. Übereinstimmung von Namen dar. Damit rückt die Objektkern-Notation optisch und konzeptionell in die Nähe von Harel's Statecharts [Harel 88].

\subsection{Klassen}

Wesentliches Element des Objektkems ist die Definition von Klassen. Die Darstellungen von Klassen bzw. Objekten sind flächig, damit sie die lokalen Komponenten (Attribute und Methoden) optisch umschließen können; wir verwenden Rechtecke mit abgerundeten Ecken. In der oberen Seite des Rechtecks integriert steht der Name der dargestellten Klasse. So repräsentiert das unten rechts gezeigte Rechteck einen Behälter für die Klassenstruktur der Klasse Eintragung. In dem Behälter kann man weitere Behälter unterbringen, welche die Attribute (siehe Abschnitt 2.2) der Klasse bezeichnen; außerdem werden die Methoden als in den Klassen enthalten dokumentiert (siehe Abschnitt 2.4). Wesentlich bei dieser graphischen Modellierung ist die optisch deutliche Unterscheidung zwischen als Teilen enthaltenen Datentypen und Datentypen, auf die nur verwiesen wird.

\subsection{Attribute}

In der Abbildung rechts erkennt man, daß Eintragung zwei Attribute enthält: einen Namen vom Typ STRING und eine Nummer vom Typ INTEGER. Die Bezeichner von Typen, die in den Kästen stehen, sind entweder die

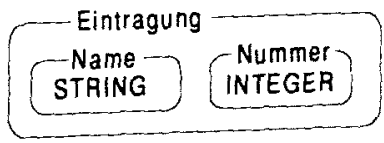
Namen von Basistypen wie STRING, INTEGER, REAL, BOOLEAN oder aber die Namen anderer, selbstdefinierter Klassen.

Als speziellen Basistyp gibt es auch den Pointer auf andere Datentypen. Dieser wird durch einen gestrichtelten Pfeil mit "dickem" Startpunkt dargestellt. In der Abbildung links wird der Typ Indizierte_Eintragung gezeigt, in dem ein Verweis auf Eintragung enthalten ist - Eintragung ist nicht Teil der Objekte vom Typ Indizierte Eintragung. Außerdem können strukturierte Datentypen eingetragen Indizierte_Eintragung ___ werden, wie etwa Felder oder Mengen; Verbünde sind

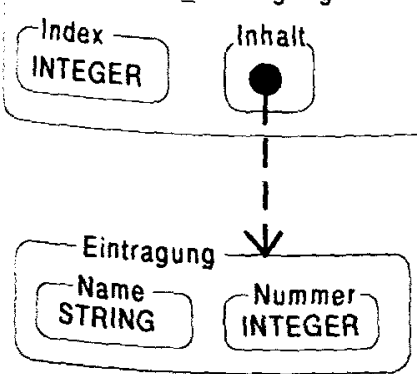
ausdrückbar. 


\subsection{Vererbung}

Vererbungsbeziehungen zwischen Klassen drückt man durch einen "erbt-von"-Pfeil aus, der von der erbenden zur vererbenden Klasse führt. Durch diese Richtung zeigt der Pfeil die Richtung an, in der man von einer angesprochenen Klasse aus ein ererbtes Atrribut bzw. eine ererbte Methode suchen muß. Um den Mehrdeutigkeiten multipler Vererbung aus dem Weg zu gehen, sei hier nur einfache Vererbung erlaubt; das heißt, von jeder Klasse darf höchstens ein Vererbungspfeil ausgehen. Die Abbildung rechts zeigt

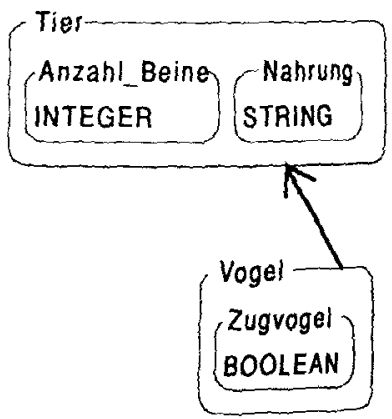
die Vererbungsbeziehung zwischen den Klassen Tier und Vogel. Ein Objekt vom Typ Vogel hat das Attribut Zugvogel, aber auch Anzahl Beine und Nahrung. Zur Vermeidung der Überfrachtung von Bildern werden die ererbten Attribute (und später auch Methoden) nicht wiederholt. Nur wenn Methoden oder Attribute einer vererbenden Klasse auf tieferer Ebene überschrieben werden, wiederholt man die Symbole in den spezielleren Klassen.

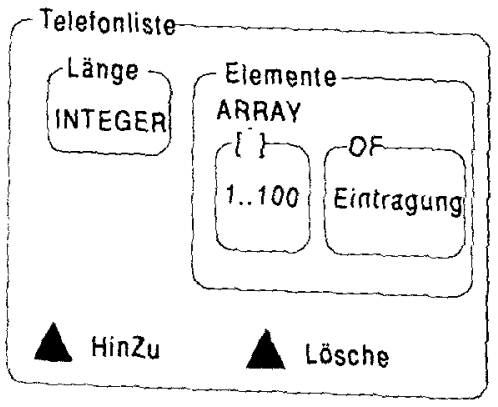

werden. Dies ist zunächst eine rein empirische Beobachtung, die im Einzelfall auch
unzutreffend sein kann, erweist Wir definieren kann, erweist sich aber als grundlegend vemünftige Technik.

(des Dreiecks) mit Parameter für eine Methode durch Verbindung des Methodensymbols einerseits die Deklaration Typkonstrukt eines Attributs eines Objektes. Dies bewirkt Attributs. Andererseits des Parametertyps - nämlich mit dem Typ des angebundenen Parameters. Die Rits dokumentien die Verbindung die vorgeschene Verwendung des Methode heraus an und ung der Pfeile zeigt den DatenfluB in die Methode oder aus der gewünscht, kann man gestricheidet auf diese Weise Eingabe- und Ausgabeparameter. Falls zu den gestrichelten Pfeileneite und durchgezogene Pfeile unterscheiden, um - in Analogie (gestrichelt) und durch Wertkei Zeigern - zwischen der Parameterübergabe über Referenzen Die Abbildung auf der nakopie (durchgezogen) zu unterscheiden.

Lösche mit ihmen Fingabehsten Seite zeigt die Spezifikation der Methoden Hinzu und Der Parameter

Da Lösche eine Eis von Lösche ist ein INTEGER-Wert im Unterbereich 1.100. dem Index klar Eestragung der Telefonliste löschen soll, ist durch die Verbindung mit spezifiziert. 
Die Methode Hinzu soll ein neues Paar (Name, Nummer), also eine neue Eintragung erzeugen. Somit sind die übergebenen Parameter Wer und WelcheNr die entsprechenden Attributwerte der neuen Eintragung.

Diese Art der Spezifikation von Parametern kann man auch durch den Zweck der Spezifikation des Objektkems rechtfertigen, der die für die Benutzung relevanten Objekte und Attribute festlegen soll: In der fertigen Applikation muß der Benutzer die Parameter angeben; daher müssen sie im Objektkern entweder direkt als Daten auftreten, oder aber es handelt sich um reine Steuerinformationen über den vom Benutzer zu steuernden Prozeß. Wie man im zweiten Fall verfahren kann, zeigt die folgende Erweiterung unseres Beispiels.

Wenn wir zum Beispiel die Methode Hinzu dahingehend erweitern wollen, daß sie einen BOOLEAN-Wert zurückgibt, der das korrekte Ausführen widerspiegelt (z.B. true genau dann,

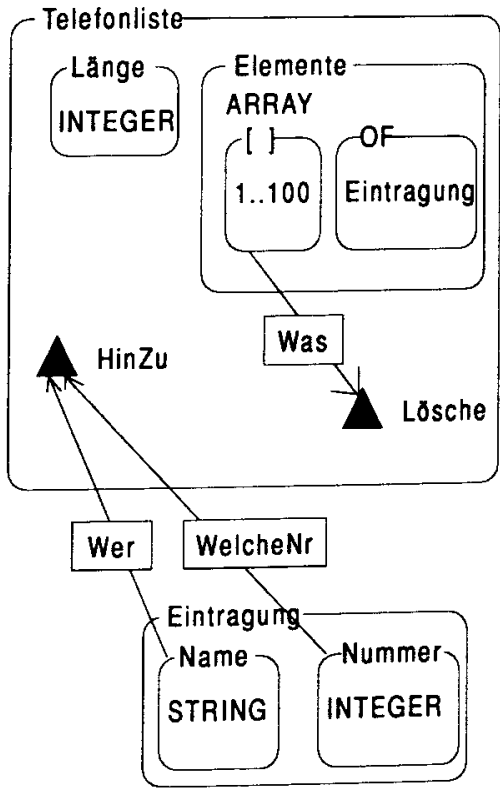
wenn die Methode korrekt ausgeführt wurde), so stehen wir vor dem Problem, daB sich kein Attribut kanonisch anbietet, um diese Information aufzunehmen. Die Lösung dafür ist, ein zunächst künstlich erscheinendes Objekt mit geeigneten Attributen einzuführen.

Reflektiert man den Zweck der Spezifikation des Objektkerns als Festlegung der für den Benutzer relevanten Komponenten eines Systems, so ist die eingeführte Objektart Rückmeldung keineswegs so künstlich, wie es zuerst schien. Die Festlegung, daß eine Benutzerfunktion eine Ausgabe erzeugt, macht nur dann Sinn, wenn diese intern erzeugte Information dem Benutzer auch in irgendeiner Form vermittelt wird. Somit repräsentieren Objektarten wie die gezeigte Rückmeldung Objekte der

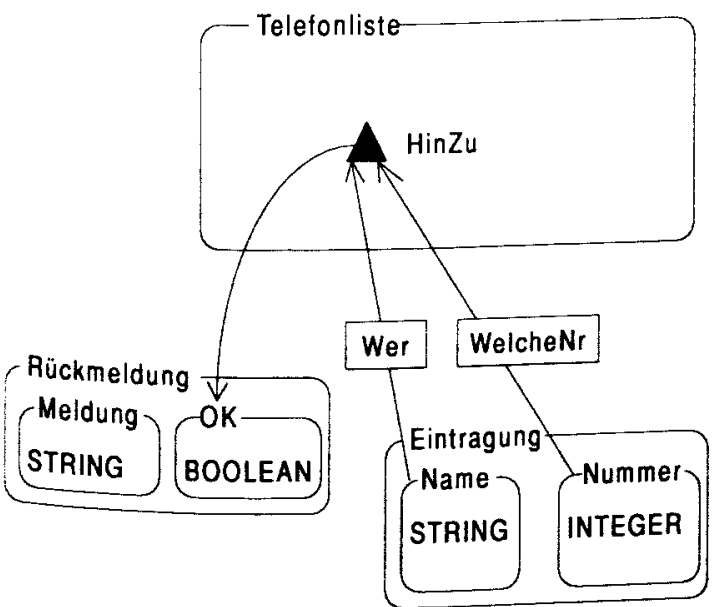
Benutzungsschnittstelle, deren sich auch die Anwendung "bewuBt" sein muß, da sie sie für Mitteilungen an den Benutzer benötigt.

\section{Methodischer Einsatz der Objektkern-Notation}

Die Notation, wie im vorigen Kapitel eingeführt, bildet den Kern einer Entwicklungsmethodik von Benutzungsschnittstellen objektorientierter Applikationen. Dabei liegt die Betonung für den Einsatz dieser Notation bei der Definition der 
Schnittstelle zwischen Anwendung und Benutzungsschnittstelle unter dem Konzept der "gemeinsamen Objekte". Die Ermittlung der Objektkern-Struktur ist, wie die Ermittlung der Objektstruktur einer Anwendung überhaupt, ein im allgemeinen nicht-triviales Problem, dessen Behandlung unter dem Begriff objektorientierte Analyse zusammengefaßt wird. Unter der Überschrift "Methodischer Einsatz" soll es aber hier nicht um eine analytische Methode gehen, sondern darum, inwieweit eine Objektkern-Spezifikation als technisches Dokument Ausgangspunkt der anschließenden Software-Entwicklung sein kann.

\subsection{Entwicklung der Applikation: Der Benutzer als Objekt}

Ziel dieser Methodik in Bezug auf die Anwendungsentwicklung ist eine hochgradige, wenn nicht völlige Entlastung dieses Prozesses von Betrachtung von Aspekten der Benutzungsschnittstelle. Das objektorientierte Paradigma bietet hier einen naheliegenden Ansatz: Für die Anwendung ist es praktisch, den Benutzer als Objekt im Software-Sinne zu betrachten'. Unter diesem Denkansatz ist der Benutzer aus Sicht der Anwendung ein Objekt mit Attributen und Methoden. Daher ist dieses Objekt in der Lage, genau wie "echte" Software-Objekte, Methoden aufzurufen und Methoden zur Verfügung zu stellen. Die Art der Einbindung von Methoden und ihre Versorgung mit Parametern in der ObjektkernNotation erlaubt schon sehr früh und auf abstraktem Niveau eine Kontrolle darüber, welche Informationen der Benutzer später sehen wird und muB, um die ihm angebotenen Methoden korrekt einsetzen zu können.

Dadurch ist nach Festlegen des Objektkerns die Vorgehensweise für den Anwendungsentwickler in Bezug auf die Bemutzungsschnittstelle sehr klar vorgezeichnet: Seine Aufgabe besteht in der korrekten Erstellung und Verwaltung der spezifizierten Objektwelt mit den angegebenen Atrributen und Methoden. Aspekte der optischen Darstellung der Objektwelt, Interaktionstechniken zum Aufruf der Methoden und genauere Festlegung der Ausgaben an den Benutzer sind nicht seine Aufgabe. Gleichzeitig ist der Aufsatzpunkt fir den Entwickler der Schnittstelle, der dem Benutzer all diese "Dienste" anbieten muß, wohldefiniert.

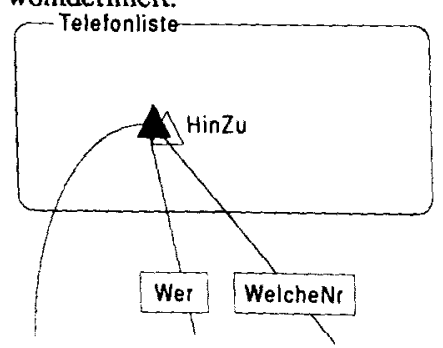

Da der Benutzer keine Software-Komponente darstellt, die der Entwickler der Anwendung kontrolliert, muß es ihm möglich sein, den Aufruf von Methoden durch den Benutzer einzuschränken. Der Entwickler weiß . aufgrund der Struktur der Anwendung - daß es nicht fir jede Methode zu jeder Zeit Sinn macht, dem Benutzer zur Verfügung zu stehen. Ähnliche Abhängigkeiten existieren für alle Methoden in dem objektorientierten System, werden aber - durch "korrekte" Verwendung von Methoden durch andere Systemteile - implizit beachtet. Es ist daher sinnvoll, den dem Benutzer zur Verfügung stehenden Methoden, von seiten der Anwendung eine APPLICABLE-Funktion zuzuordnen: Diese liefert einen Booleschen Wert true genau dann, wenn die Methode anwendbar ist und false sonst. Die Entscheidung über die Anwendbarkeit sollte ein Objekt selbst steuern, daher benötigt eine APPLICABLEFunktion keine Eingabeparameter. Da auch das Format und die Verwendung der Ausgabe implizit vordefiniert sind, ist auch keine optische Verbindung dafür im eigentlichen Objektkem erforderlich. Optisch kennzeichnen wir das Vorhandensein einer APPIICABIE-

\footnotetext{
Interessanterweise erscheint det Benutzer in Kontext von objektorientiener Analyse durchans noch als "Objekt"; im endguhizes

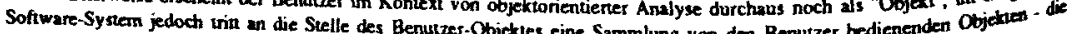
Benuraungsschnirtstle
} 
Funktion durch Unterlegen des "Methoden-Dreiecks" mit einem weißen "Schatten", wie oben links gezeigt. Nicht jede Methode muß eine zugeordnete APPLICABLE-Funktion besitzen.

\subsection{Entwicklung der Benutzungsschnittstelle}

Prinzipiell geht der BS-Entwickler genauso vor, wie der Entwickler der eigentlichen Anwendung: Er erweitert den Objektkern um weitere Klassen, Attribute und Methoden. Ziel dieser Ergänzungen ist die Gestaltung der Benutzungsschnittstelle mit den Aspekten graphische Darstellung der Objektwelt und Erkennen und Bearbeiten von Eingaben des Benutzers. Dieser Gestaltungsproze $B$ ist durch eine Dualität der Betrachtungsweise gekennzeichnet: Auf der einen Seite müssen die bildliche Ausgabe und die Interaktionstechniken in ihrer Erscheinungsform "nach außen", also wie für den Benutzer erkennbar, beschrieben werden; andererseits müssen die Ableitung der graphischen Darstellung aus den Objekten bzw. die Wirkung der Interaktion "nach innen", auf die intemen Objekte, definiert werden. In der Folge sprechen wie jeweils von der externen und der internen Sicht auf die Darstellung bzw. die Interaktionen. Technisch geht der BS-Entwickler so vor, daß er die graphische Ausgabe durch Hinzufügen graphischer Attribute und die Interaktion durch Hinzufügen von Interaktionsmethoden definiert.

\section{Das Werkzeug}

Eine experimentelle Version eines die Objektkern-Notation unterstützenden Werkzeugs wird zur Zeit implementiert. Dabei steht zunächst die reine Editorfunktion im Vordergrund. Für die beiden folgenden getrennten Entwicklungen von Anwendung und Benutzungsschnittstelle sind eine Reihe sehr mächtiger Anschlüsse denkbar. Zentral ist für beide Bereiche, daß das Werkzeug Mechanismen anbieten muß, um Teile des Diagramms "einzufrieren", um die Konsistenz des Objektkerns zu garantieren. Endziel wäre eine netzwerkgestützte Mehrbenutzer-Version eines solchen Editors .

\section{Literatur}

Booch, G

Object Oriented Design with

Applications, Benjamin/Cummings,

Redwood City, CA, 1991

Coad, P; Yourdon, E

Object-Oriented Analysi, Prentice-

Hall, Englewood Cliffs, 1991

Dance, J R; Granor, T E; Hill, R D;

Hudson, S E; Meads, J; Myers, B A;

Schulert, A

The Run-time Structure of UIMS-

Supported Applications, Computer

Felser, W

Graphics 21, 2, 1987

Kreativer objektorientierter Entwurf:

Methodik, Diplomarbeit, Universität

- GH - Paderborn, Fachbereich

Harel, D

Mathematik/Informatik, August 1992

On Visual Formalisms, CACM, Vol.

31, No. 5, 1988.
Pfaff, G E

User Interface Management Systems:

Proceedings of the Seeheim

Workshop, Springer-Verlag, Berlin,

1985

Rubin, K S; Goldberg, A Object Behavior Analysis, CACM

Vol. 35, No. 9, S.48-62, 1992

Szekely, P; Myers, B A

A User Interface Toolkit Based on

Graphical Objects and Constraints, OOPSLA'88, SIGPLAN Notices,

November 1988

Wirfs-Brock, R; Wilkerson, B; Wiener, L

Designing Object-Oriented Software,

Prentice-Hall, Englewood Cliffs,

1990 